\title{
PAPER \\ Resource Block Assignment to Reduce Peak to Average Power Ratio in Multi-User OFDM*
}

\author{
Osamu TAKYU ${ }^{\dagger a)}$, Yohtaro UMEDA ${ }^{\dagger \dagger}$, Members, Fumihito SASAMORI ${ }^{\dagger}$, Senior Member, \\ and Shiro HANDA ${ }^{\dagger}$, Member
}

\begin{abstract}
SUMMARY This paper proposes the assignment of resource blocks (RBs) to reduce the peak-to-average power ratio (PAPR) of orthogonal frequency division multiplexing (OFDM) in a multi-user OFDM system. This system ranks the users according to the channel state information (CSI) for $\mathrm{RB}$ assignment. In our proposed technique, an RB is assigned to either the first- or second-ranked mobile station (MS) to minimize the PAPR of the OFDM signal. While this process reduces the PAPR, the throughput is also reduced because of the user diversity gain loss. A PAPR-throughput tradeoff is then established. Theoretical analyses and computer simulations confirm that when the number of MSs becomes large, the PAPR-throughput tradeoff is eased because of the minimal effect of the diversity gain loss. Therefore, significant PAPR reduction is achieved with only a slight degradation in the throughput.

key words: multiuser OFDM, PAPR, resource block assignment
\end{abstract}

\section{Introduction}

Multiuser orthogonal frequency division multiplexing (OFDM) systems achieve high throughput through the significant user diversity gain realized by adaptive resource block (RB) assignment on the basis of channel state information (CSI) [1]. Unfortunately, OFDM signals suffer from high peak-to-average power ratios (PAPRs), which significantly degrade the efficiency of the power amplifiers because of the large input back off levels needed [2].

To reduce the PAPR, various techniques have been proposed [3]-[8]. These techniques are mainly categorized into nonlinear and linear processes. In the nonlinear process such as clipping and filtering [3] and companding [4], the high peak power of a signal is directly suppressed. Even though the PAPR reduction can be significant, the error rate performance due to nonlinear distortion is significantly degraded. Nonlinear distortion is a serious problem in high-speed communication because high-order modulation schemes are highly vulnerable to nonlinear distortion. In the linear process such as the partial transmit sequence (PTS) [5], coding [6], and selected mapping (SLM) [7],

\footnotetext{
Manuscript received September 17, 2013.

Manuscript revised March 16, 2014.

${ }^{\dagger}$ The authors are with the Department of Electrical \& Electronic Engineering, Shinshu University, Nagano-shi, 380-8553 Japan.

${ }^{\dagger \dagger}$ The author is with the Department of Electrical Engineering, Tokyo University of Science, Noda-shi, 278-8510 Japan.

${ }^{*}$ This paper was presented in part at the IEEE ICCS 2010 Nov. 2010, IWDN2012 conjunction with IEEE PIMRC 2012, Sept. 2012, and Trisai2012, Sept. 2012.

a)E-mail: takyu@ shinshu-u.ac.jp

DOI: 10.1587/transcom.E97.B.1689
}

[8], the phase and amplitude components of each subcarrier are adjusted as the PAPR becomes small. Although nonlinear distortion can be avoided, the transmitter should pass the information of the adjusted phase and amplitude components to the receiver through a side information channel. Therefore, the frequency utilization efficiency is degraded. In addition, the receiver requires an additional process to remove the adjusted phase and amplitude components. We summarize the weaknesses of the conventional PAPR reduction techniques as follows: 1) degraded throughputs and error rates, 2) need for side information channel, and 3) additional receiver processes. A novel PAPR reduction scheme that can avoid these weaknesses is necessary.

The present paper proposes an RB assignment technique to reduce the PAPR of OFDM signals in a multiuser OFDM system. The proposed technique ranks the users according to the CSI in determining the RB assignment. If the maximum carrier-to-noise-power ratio (CNR) criterion is used for the RB assignment [9], mobile stations (MSs) with higher instantaneous CNRs have higher rank. The conventional multiuser OFDM systems assign the RB to the firstranked MS. However, the proposed technique assigns some RBs as well as the other RBs to the first- and second-ranked MSs, as the PAPR becomes small. To determine the suitable $\mathrm{RB}$ assignment, the transmitter constructs some assignment candidates, and one candidate is selected following the minimum PAPR manner. As the more RBs are assigned to the second-ranked MSs, the more assignment candidates can be constructed. As a result, more suitable assignment candidates that create smaller PAPR can be selected. Because our proposed technique sacrifices the user diversity gain, the throughput is reduced. Therefore, our proposed technique must deal with a PAPR-throughput tradeoff. The advantages of the proposed technique are as follows:

1. Because the conventional multiuser OFDM systems already use a side information channel to pass the RB assignment information to all MSs and CSI feedback, the proposed technique imposes no additional transmission overhead. If the PTS or SLM is applied to a multiuser OFDM system, side information not only for the multiuser OFDM system but also for the PTS or SLM is necessary. In a multiuser OFDM system, the total amount of side information of our proposed technique is less than that of the PTS or SLM.

2. Minimal effect on the diversity gain loss [10], [11]: 
even if many RBs are assigned to the second-ranked MSs, the degradation in the throughput is small.

3. The receiver selectively detects the $\mathrm{RB}$ in accordance with the assignment information. Because the multiuser OFDM receivers already perform this process, the proposed technique imposes no additional processing overhead on the receiver.

Owing to the first and third advantages outlined above, only the transmitter needs to be modified to implement the proposed technique. For the downlink of cellular systems, only the base station (BS) should be modified and not the MS. Therefore, the cost of implementing the proposed technique is minimal. Because the PAPR-throughput tradeoff is eased by the proposed technique (second feature), PAPR reduction is achieved with only a slight drop in the throughput. This result is confirmed by theoretical analyses and computer simulations.

The remainder of this paper is organized as follows: Sect. 2 shows the configuration of the target system, Sect. 3 explains our proposed RB assignment for PAPR reduction, Sect. 4 shows the theoretical analysis of the PAPR and the throughput, and Sect. 5 presents the numerical results that validate the effect of our proposed technique. Finally, Sect. 6 presents the conclusions and future work.

\section{System Configuration}

Figure 1 shows the system model of a multiuser OFDM system. In Fig. 1(a), we consider a cellular system and assume one BS that supports multiple MSs. We pay attention to the downlink wireless access (from BS to MS). In Fig. 1(b), the BS constructs multiple subcarriers by OFDM modulation, and RBs (composed of multiple continuous subcarriers) are assigned to the MSs for downlink transmission, where $N_{D}$ is the number of subcarriers per RB and $N_{M}$ is the number of RBs.

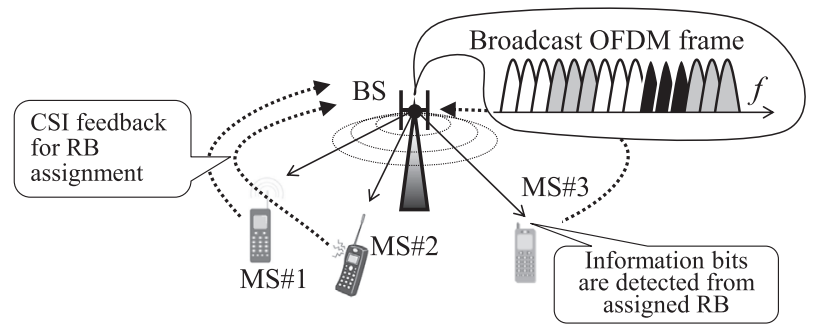

(a) Down link access and CSI feedback

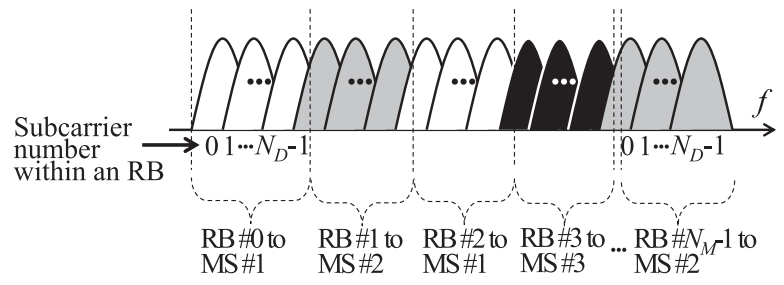

(b) RB in OFDM frame and RB's assignment

Fig. 1 Multiuser OFDM for a downlink cellular system.
Each MS periodically communicates its CSI to the BS. The BS ranks the users according to the CSI. In this study, because the maximum CNR criterion [9] is used, a large instantaneous CNR yields a high rank. We note that the other criterion such as proportional fairness can also be used in the proposed system.

The basic idea is for the BS to assign RBs to the MSs with the best downlink performance, which yields a large CNR. After assigning the RBs to the MSs, a suitable modulation and coding set (MCS) is selected. After the information bits at each MS are modulated for the subcarrier of the assigned RB, all RBs are modulated by inverse fast Fourier transform (IFFT) to yield the OFDM symbol. In this study, when a continuous series of OFDM symbols is considered as an OFDM frame, the RB assignment is not changed during one OFDM frame, where the number of OFDM symbols per OFDM frame is $N_{Q}$. The BS broadcasts the constructed OFDM frame to all the MSs, and each MS then detects the information from the assigned RBs by FFT and demodulation.

\section{Proposed PAPR Reduction Technique}

\subsection{Conventional RB Assignment}

Figure 2(a) shows that the RB is assigned to the first-ranked MS. Because the RB assignment does not consider any PAPR, a large PAPR appears with high probabilities.

\subsection{Proposed RB Assignment}

We propose an RB assignment that reduces the PAPR of the OFDM signal. Figure 2(b) shows how the proposal selects the RB assignment candidate. Figure 3 shows the process flow.

First, we rank the MSs according to the CSI feedback
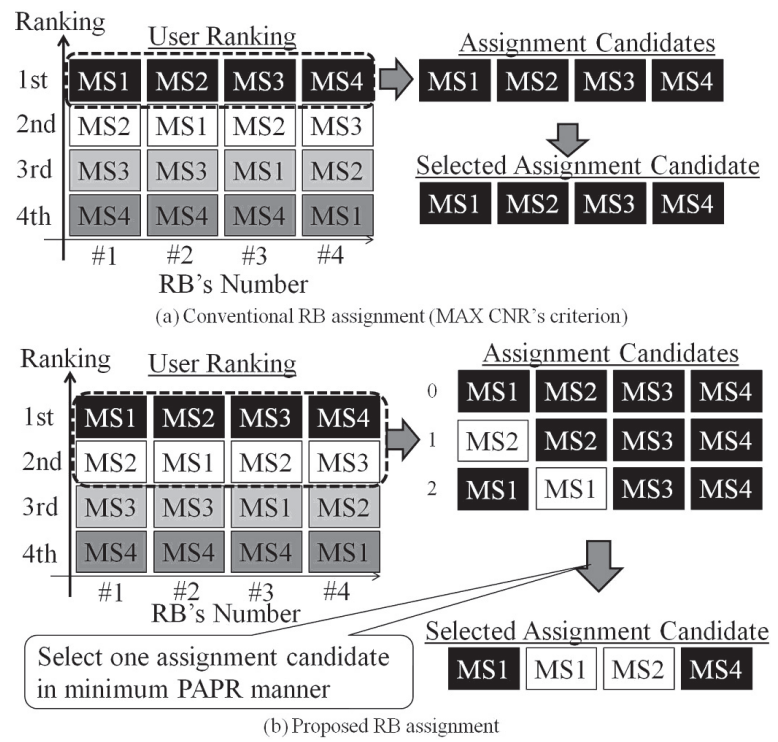

Fig. 2 RB assignment. 


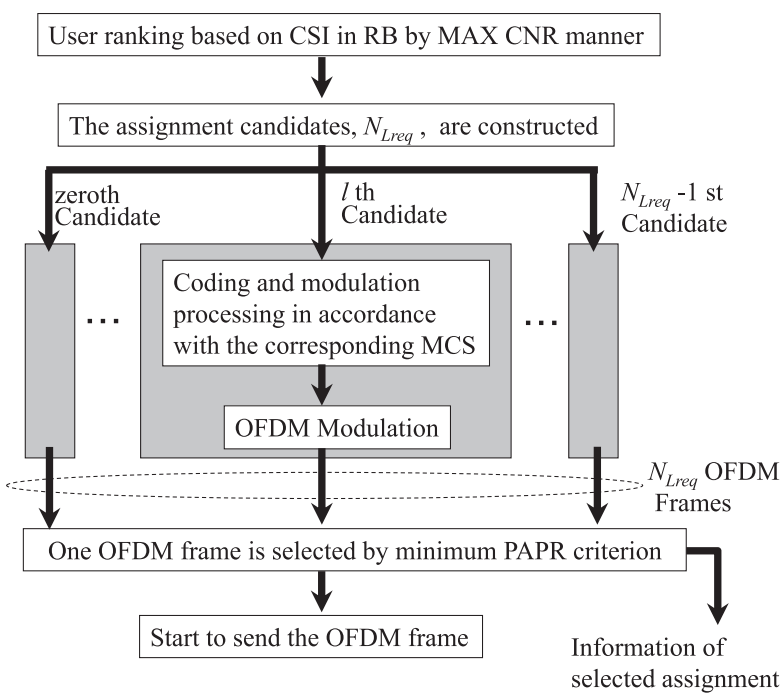

Fig. 3 Process flow of the RB assignment proposal.

as per conventional RB assignment. Second, we establish various $\mathrm{RB}$ assignment candidates. The zeroth assignment candidate assigns all RBs to the first-ranked MSs following the conventional approach. In the other candidates, the RBs are assigned to a mixture of the first- and second-ranked MSs. The total number of candidates, $N_{L \text { max }}$, is given by

$$
N_{L \max }=\sum_{k=0}^{N_{K 0}}\left(\begin{array}{c}
N_{M} \\
k
\end{array}\right),
$$

where $N_{K 0}$ is the maximum number of RBs assigned to the second-ranked MSs $\left(N_{K 0} \leq N_{M}\right)$. When $N_{K 0}$ increases, $N_{L \max }$ dramatically increases. As a result, $N_{L \max }$ may be significantly greater than that required by the $\mathrm{BS}$, where the number of candidates required by the BS to meet the desired PAPR level is $N_{\text {Lreq }}$. Therefore, we select $N_{\text {Lreq }}$ candidates among $N_{L \max }$ ones. First, we define $N_{K}$ as the minimum number of $N_{K 0}$ to $N_{L \max }$, which is greater than $N_{\text {Lreq }}$. Therefore, the following equation is stood.

$$
N_{K}=\min \left\{N_{K 0} \mid N_{L \max } \geq N_{\text {Lreq }}\right\}
$$

When the RBs assigned to the second-ranked MSs are fewer than $N_{K}$, all constructed assignments are considered as candidates. Second, when $N_{K}$ RBs are assigned to the secondranked MS, we obtain additional assignment candidates. The required assignment candidates are selected from the additional constructed assignments in a maximal total CNR manner, where the sum of the achieved CNRs for all the RBs is considered as the total CNR. Figure 4 shows an example of the assignment selection for $N_{\text {Lreq }}=7$ and $N_{M}=4$.

In each assignment candidate, the most suitable MCS is selected for each RB. When the RB assignment is decided by a unit of one OFDM frame in the considered multiuser OFDM system, the OFDM symbols of one OFDM frame are constructed by IFFT. This process yields $N_{\text {Lreq }}$ OFDM frames. Because all the RBs are combined by IFFT, if any one $\mathrm{RB}$ assignment is changed, the envelope of the OFDM

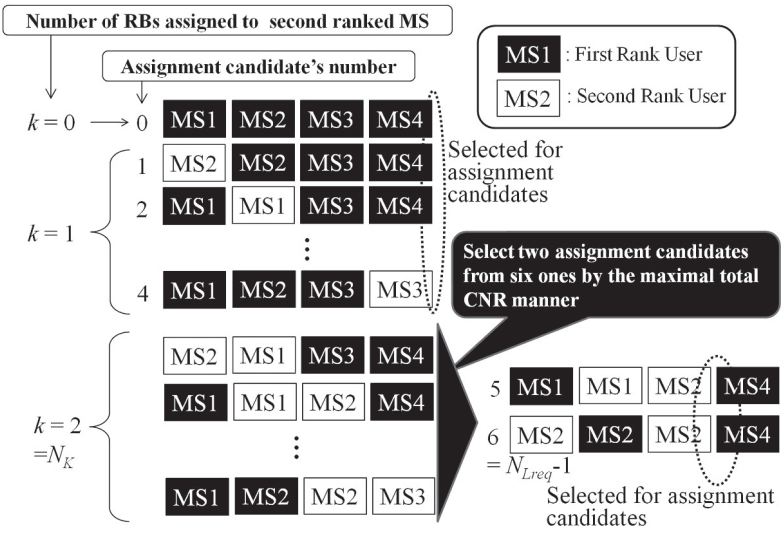

Fig. 4 Example of the selection for assignment candidates for $N_{\text {Lreq }}=7$ and $N_{M}=4$

frame is changed, which is similar to the PTS [5]. Therefore, the BS uses the minimum PAPR criterion to select one OFDM frame. When the selected OFDM frame is broadcast to all the MSs, the assignment selected by the BS should be communicated to all MSs through the side information channel, similar to that done in the conventional approach.

In the proposed technique, one assignment candidate is selected in a minimum PAPR manner following the SLM technique [7]. As the number of candidates increases, the PAPR reduction is strengthened [7]. However, in our proposed technique, to increase the number of candidates, the number of RBs assigned to the second-ranked MSs should be large. Because the achieved CNR becomes smaller, a lower level MCS set should be selected to meet communication reliability. Therefore, the throughput is reduced, which results in a PAPR-throughput tradeoff. We can consider that the RB is assigned to the third- and fourth-ranked MSs to reduce the PAPR. In this study, only the second-ranked MSs are considered for simplicity.

\subsection{Revising the PAPR Criterion}

Because the proposed technique evaluates the PAPR of all OFDMs in one frame to decide the RB assignment, we introduce the frame PAPR [12].

The PAPR per OFDM symbol is a well-known criterion [3], and the definition of the PAPR for the $q$ th OFDM symbol $\left(q \in 0,1, \ldots, N_{Q}-1\right)$ is as follows:

$$
\operatorname{PAPR}_{q}=\xi_{q}=\max _{0 \leq u<U-1} \frac{\left|s_{q}[u]\right|^{2}}{P_{\text {avg }}}
$$

where $s_{q}[u]$ is the $u$ th sample in the $q$ th OFDM symbol, $P_{\text {avg }}$ is the average power of the OFDM symbol, and $U$ is the total number of samples in the OFDM symbol. In this study, the PAPR in Eq. (3) is referred to as symbol PAPR.

The frame PAPR is defined as

$$
\text { Frame PAPR }=\Xi=\max _{q \in 0,1, \ldots N_{Q^{-1}}} \operatorname{PAPR}_{q} .
$$

Because the symbol PAPRs of all OFDM symbols in an 
OFDM frame are smaller than or equal to the frame PAPR, the BS can find the assignment that offers the smallest frame PAPR; thus, the symbol PAPRs of all OFDM symbols in the OFDM frame are small.

\subsection{Modifying the Selection of Assignment Candidate for} Nonlinear Power Amplifier

In a practical wireless system, when the power amplifier of an RF front end is specified, the required PAPR to satisfy the acceptable out-of-band emission of an emitted signal is determined. Because the PAPR reduction over the required one is meaningless, we modify the selection of the assignment candidate in the proposed technique.

We set the target PAPR, $\Xi_{t h}$. When all RBs are assigned to the first-ranked MS, the frame PAPR of the OFDM frame is evaluated. If it is smaller than $\Xi_{t h}$, this OFDM frame is selected. Otherwise, the number of RBs assigned to the second-ranked MS is increased by one. Because this process yields multiple assignment candidates, the OFDM frames of the newly constructed assignment candidates are modulated, and their frame PAPRs are evaluated. If the frame PAPR of the OFDM frame is smaller than $\Xi_{t h}$, the OFDM frame is selected; otherwise, the number of RBs assigned to the second-ranked MS is increased by one RB. The process is repeated until the frame- PAPR is smaller than $\Xi_{t h}$. If the frame PAPRs of the $N_{\text {Lreq }}$ OFDM frames are not smaller than $\Xi_{t h}$, the OFDM frame with the smallest frame PAPR is selected.

This modification avoids excessive assignment to the second-ranked MSs. Therefore, large throughput is maintained. Once the frame PAPR of an OFDM frame meets the target, the PAPR evaluation process is stopped. Therefore, the computational complexity of the transmitter is reduced.

\section{Theoretical Analyses of the Throughput and PAPR}

We derive the throughput and PAPR by theoretical analyses. It is difficult to analyze the actual wireless environment. Instead, we use a simplified system model. These results clarify the trend of the proposed technique. In the following subsections, we detail the assumptions made.

In our proposed technique, the cumulative distribution function $(\mathrm{CDF})$ of throughput is defined as follows:

$$
\begin{aligned}
& P_{\text {pro }}\left(R<R_{O}\right)\left(N_{\text {Lreq }}\right) \\
& \quad=r_{1 s t}\left(N_{\text {Lreq }}\right) P_{1}\left(R<R_{O}\right)+r_{2 n d}\left(N_{\text {Lreq }}\right) P_{2}\left(R<R_{O}\right) .
\end{aligned}
$$

where $r_{1 s t}\left(N_{\text {Lreq }}\right)$ and $r_{2 n d}\left(N_{\text {Lreq }}\right)$ are the probabilities of RB assignment to the first- and second-ranked MSs, respectively when the number of assignment candidates is $N_{\text {Lreq }}$, and $P_{1}\left(R<R_{O}\right)$ and $P_{2}\left(R<R_{O}\right)$ are the outage probability of the normalized throughput, $R$, for the first- and secondranked MSs, respectively. In the following subsection, we show how to derive each term.
4.1 Throughput Analysis for the First- and Second-ranked MS

We assume the full buffering model, and thus, each user is continually sent traffic. Rayleigh fading channels are considered, where the fading within each RB is constant, but the fading of the different RBs follows the stochastic model with independent and identical distribution (i.i.d.). In the following analysis, because we pay attention to one RB, the RB index is omitted. Because of the i.i.d. model assumption, the result of the analysis can be equally applied to each RB.

The instantaneous received CNR of the $n$th MS is $\gamma_{n} \Gamma_{n}$, where $\gamma_{n}$ is the square component of the Rayleigh fading and $\Gamma_{n}$ is the average CNR. $\gamma_{n}$ is a random variable with exponential distribution

$$
f\left(\gamma_{n}\right)=e^{-\gamma_{n}} .
$$

If the transmitted signal uses an infinite-length code sequence, the channel capacity of the additive white Gaussian noise (AWGN) channel is decided by Shannon's information theory [13]. If the fading coefficient is given, the fading channel is considered to be an AWGN channel whose signal power is decided by the fading coefficient [14]. Therefore, in this study, when the fading coefficient is given, we consider that the channel capacity of the fading channel is also determined by Shannon's information theory.

From [3], we derive the channel capacity per subcarrier in an OFDM system, where the average range of the frequency domain is equal to the frequency bandwidth of the RB. When the number of subcarriers per RB is $N_{D}$ and the frequency bandwidth of the subcarrier is $\Delta f$, the frequency bandwidth of the RB is $\Delta f N_{D}$. Therefore, the channel capacity of an RB assigned to the $n$th MS, namely $C_{n}$, is derived as follows:

$$
\begin{aligned}
C_{n} & =\Delta f N_{D} \cdot \frac{1}{N_{D}} \sum_{i=0}^{N_{D}-1} \log _{2}\left(1+\gamma_{n} \Gamma_{n}\right) \\
& \left.=\Delta f N_{D} \log _{2}\left(1+\gamma_{n} \Gamma_{n}\right) \quad \text { (bits } / \text { sec }\right) .
\end{aligned}
$$

Because the inverse of $\Delta f$ is equal to the time duration of the OFDM symbol, the throughput for a unit time duration of one OFDM symbol is $C_{n} / \Delta f$. When it is divided by the number of subcarriers $N_{D}$, the normalized throughput per OFDM symbol per subcarrier is defined as

$$
\begin{aligned}
R_{n} & =\frac{C_{n}}{\Delta f N_{D}} \\
& =\log _{2}\left(1+\gamma_{n} \Gamma_{n}\right) \text { (bits/symbol/subcarrier). }
\end{aligned}
$$

If the information signal uses a finite-length code, the normalized throughput is smaller than that in Eq. (8). Although an information signal with a finite-length code is more practical, the difference between the practical throughput and that in Eq. (8) is ignored to simplify the analysis.

When the $n$th MS is assigned to the first rank 


$$
\gamma_{k}<\frac{\gamma_{n} \Gamma_{n}}{\Gamma_{k}} \quad(k \in 0,1, \ldots N-1 \backslash n),
$$

where $N$ is the number of MSs and $\backslash$ indicates the exception operator. Therefore, $k=0,1, \ldots, N-1 \backslash n$ indicates that $k$ takes $0,1, \ldots, N-1$, except for $n$. When $\gamma_{n}$ is given, except for the $n$th MS, the conditional probability that Eq. (9) is satisfied for all MSs is

$$
\begin{gathered}
P_{1}\left(\gamma_{k}<\frac{\gamma_{n} \Gamma_{n}}{\Gamma_{k}}\{\forall k \in 0,1, \ldots N-1 \backslash n\} \mid \gamma_{n}\right) \\
=\prod_{\forall k \in 0,1, \ldots, N-1 \backslash n} \int_{0}^{\frac{\gamma_{n} \Gamma_{n}}{\Gamma_{k}}} f\left(\gamma_{k}\right) d \gamma_{k} \\
=\prod_{\forall k \in 0,1, \ldots, N-1 \backslash n}\left(1-e^{-\frac{\gamma_{n} \Gamma_{n}}{\Gamma_{k}}}\right) .
\end{gathered}
$$

The occurrence probability of $\gamma_{n}, P\left(\gamma_{n}\right)$, is

$$
P\left(\gamma_{n}\right)=f\left(\gamma_{n}\right) d \gamma_{n}=e^{-\gamma_{n}} \Delta \gamma_{n},
$$

where $\Delta \gamma_{n}$ is the incremental value of $\gamma_{n}$. Therefore, the probability that the $n$th MS is assigned to the first rank, $P_{1}\left(\gamma_{n}\right)$, is the joint probability that satisfies both Eq. (9) and the occurrence of $\gamma_{n}$ and it is derived as follows:

$$
\begin{aligned}
& P_{1}\left(\gamma_{n}\right) \\
& =P_{1}\left(\gamma_{k}<\frac{\gamma_{n} \Gamma_{n}}{\Gamma_{k}}\left\{k^{\forall} \in 0,1, \ldots N-1 \backslash n\right\}, \gamma_{n}\right) \\
& =P_{1}\left(\gamma_{k}<\frac{\gamma_{n} \Gamma_{n}}{\Gamma_{k}}\left\{k^{\forall} \in 0,1, \ldots N-1 \backslash n\right\} \mid \gamma_{n}\right) \cdot P\left(\gamma_{n}\right) \\
& =e^{-\gamma_{n}} \prod_{k^{\forall} \in 0,1, \ldots N-1 \backslash n}\left(1-e^{-\frac{\gamma_{n} \Gamma_{n}}{\Gamma_{k}}}\right) \Delta \gamma_{n} .
\end{aligned}
$$

To simplify the analysis, we assume that all users have the same average CNR. We define $\Gamma_{O}=\Gamma_{0}=\ldots=\Gamma_{N-1}$. Therefore,

$$
P_{1}\left(\gamma_{n}\right)=e^{-\gamma_{n}}\left(1-e^{-\gamma_{n}}\right)^{N-1} \Delta \gamma_{n} .
$$

From this result, the outage probability of the instantaneous CNR normalized by the average CNR, namely, $P_{1}\left(\gamma_{n}<\gamma_{O}\right)$, can be derived as follows:

$$
\begin{aligned}
P_{1}\left(\gamma_{n}<\gamma_{O}\right) & =\int_{0}^{\gamma_{O}} e^{-\gamma_{n}}\left(1-e^{-\gamma_{n}}\right)^{N-1} d \gamma_{n} \\
& =\frac{1}{N}\left(1-e^{-\gamma_{O}}\right)^{N},
\end{aligned}
$$

where $\gamma_{O}$ is the fading coefficient used to define the cumulative distribution function (CDF) and in the analysis of Eq. (14), $\Delta \gamma_{n}$ is converted to the infinitesimal value of it, $d \gamma_{n}$. Similarly, the probability that the $n$th MS is assigned to the second rank, i.e. $P_{2}\left(\gamma_{n}\right)$, is

$$
\begin{aligned}
P_{2}\left(\gamma_{n}\right)= & f\left(\gamma_{n}\right) \int_{\frac{\gamma_{n} \Gamma_{n}}{\Gamma_{n_{1}}}}^{\infty} f\left(\gamma_{n_{1}}\right) d \gamma_{n_{1}} \\
& \cdot \prod_{k^{\vee} \in 0,1, \ldots, N-1 \backslash\left(n, n_{1}\right)} \int_{0}^{\frac{\gamma_{n} \Gamma_{n}}{\Gamma_{k}}} f\left(\gamma_{k}\right) d \gamma_{k} \cdot \Delta \gamma_{n}
\end{aligned}
$$

$$
=e^{-2 \gamma_{n}}\left(1-e^{-\gamma_{n}}\right)^{N-2} \Delta \gamma_{n},
$$

where $n_{1}$ is the first-ranked MS number. The outage probability, $P_{2}\left(\gamma_{n}<\gamma_{O}\right)$, is derived as follows:

$$
\begin{aligned}
& P_{2}\left(\gamma_{n}<\gamma_{O}\right)=\int_{0}^{\gamma_{O}} e^{-2 \gamma_{n}}\left(1-e^{-\gamma_{n}}\right)^{N-2} d \gamma_{n} \\
& =\frac{1}{N(N-1)}\left(1-e^{-\gamma_{O}}\right)^{N-1}\left(1+(N-1) e^{-\gamma_{O}}\right) .
\end{aligned}
$$

From the analysis of the outage probability, we can calculate the diversity order [15]. For the first-ranked MS, the diversity order, $d_{1}$, is

$$
\begin{aligned}
d_{1} & =\lim _{\gamma_{O} \rightarrow 0} \frac{\log \left(P_{1}\left(\gamma_{n}<\gamma_{O}\right)\right)}{\log \left(\gamma_{O}\right)} \\
& =\lim _{\gamma_{O} \rightarrow 0}\left[\frac{\log (1 / N)}{\log \left(\gamma_{O}\right)}+\frac{N \log \left(1-e^{-\gamma_{O}}\right)}{\log \left(\gamma_{O}\right)}\right]=N .
\end{aligned}
$$

Similarly, for the second-ranked MS, the diversity order, $d_{2}$ is

$$
\begin{aligned}
d_{2}= & \lim _{\gamma_{O} \rightarrow 0} \frac{\log \left(P_{2}\left(\gamma_{n}<\gamma_{O}\right)\right)}{\log \left(\gamma_{O}\right)} \\
= & \lim _{\gamma_{O} \rightarrow 0}\left[\frac{\log (1 /(N(N-1)))}{\log \left(\gamma_{O}\right)}+\frac{(N-1) \log \left(1-e^{-\gamma_{O}}\right)}{\log \left(\gamma_{O}\right)}\right. \\
& \left.+\frac{\log \left(1+(N-1) e^{-\gamma_{O}}\right)}{\log \left(\gamma_{O}\right)}\right]=N-1
\end{aligned}
$$

Therefore, when an RB is assigned to the second-ranked MS, we allow a reduction of one diversity order.

We calculate the outage probability of the normalized throughput. Because the number of MSs is $N$, the pattern of the first-ranked MS is $N$, and the combination of the firstand second-ranked MSs is $N(N-1)$. From Eq. (8), when

$$
\gamma_{O}=\frac{2^{R_{O}}-1}{\Gamma_{O}},
$$

the outage probability of the throughput is derived as

$$
\begin{aligned}
P_{1}\left(R<R_{O}\right)= & \left(1-e^{-\frac{2^{R_{O}-1}}{\Gamma_{O}}}\right)^{N} \\
P_{2}\left(R<R_{O}\right)= & \left(1-e^{-\frac{2^{R_{O}-1}}{\Gamma_{O}}}\right)^{N-1} \\
& \cdot\left\{1+(N-1) e^{-\frac{2^{R_{O}-1}}{\Gamma_{O}}}\right\},
\end{aligned}
$$

where $R_{O}$ is the normalized throughput used to decide the outage probability of the normalized throughput.

\subsection{RB Assignment Probabilities}

In the proposed technique, we hardly consider that the particular assignment candidate is dominantly selected for a small PAPR, but each assignment candidate is almost equally selected. When we assume that the selection of the assignment candidate follows a stochastic model with uniform distribution [0 $N_{\text {Lreq }}$ ), the RBs assigned to the first- 
and second-ranked MSs are decided by the selection probabilities.

In proposed RB assignment, when $0,1, \ldots, N_{K}-1 \mathrm{RBs}$ are assigned to the second-ranked MS, the constructed assignments are used for the candidates. When $N_{K}$ RBs are assigned to it, the constructed assignments are selectively used as meeting the required number of candidates, $N_{\text {Lreq }}$. Therefore, in the analysis of RB assignment probabilities, each case is considered, separately.

In Sects. 4.2.1 and 4.2.2, $N_{K} \geq 1$ is stood. In $N_{K}=0$, since all the RBs are assigned to the first-ranked MS, the probabilities of RB assigning the first-ranked MS and the second-ranked one are 1 and 0 , respectively.

\subsection{1 $0,1, \ldots, N_{K}-1$ RBs Assigning Second-ranked User}

When the number of RBs assigned to the second-ranked MSs is equal to or smaller than $N_{K}-1$, the number of assignment candidates is

$$
N_{L}\left(N_{K}-1\right)=\sum_{k=0}^{N_{K}-1}\left(\begin{array}{c}
N_{M} \\
k
\end{array}\right),
$$

where $k$ is equal to the number of $\mathrm{RBs}$ assigned to the second-ranked MS. After one RB is assigned to the firstranked MS, the total numbers of assignment candidates, $N_{L 1 s t}\left(N_{k}-1\right)$, is derived as follows:

$$
N_{L 1 s t}\left(N_{K}-1\right)=\sum_{k=0}^{N_{K}-1}\left(\begin{array}{c}
N_{M}-1 \\
k
\end{array}\right) \quad\left(N_{K} \geq 1\right)
$$

Similarly, after one RB is assigned to the second-ranked MS, the numbers of assignment candidates, $N_{L 2 n d}\left(N_{K}-1\right)$ is also derived as follows:

$$
\begin{aligned}
N_{L 2 n d} & \left(N_{K}-1\right) \\
= & \left\{\begin{array}{l}
0 \quad\left(N_{K}=1\right) \\
\sum_{k=0}^{N_{K}-2}\left(\begin{array}{c}
N_{M}-1 \\
k
\end{array}\right) \quad\left(N_{K} \geq 2\right) .
\end{array}\right.
\end{aligned}
$$

\subsection{2 $N_{K}$ RBs Assigning Second-ranked User}

When $N_{K}$ RBs are assigned to the second-ranked MS, the number of additional constructed assignment candidates is

$$
N_{L}^{\prime}\left(N_{K}\right)=\left(\begin{array}{c}
N_{M} \\
N_{K}
\end{array}\right) .
$$

After one RB is assigned to the first-ranked MS and another $\mathrm{RB}$ is assigned to the second-ranked MS, the $N_{L}^{\prime}\left(N_{K}\right)$ assignments are categorized into L1st and L2nd categories. The numbers of each category assignment $N_{L 1 s t}^{\prime}$ and $N_{L 2 n d}^{\prime}$ are

$$
\begin{aligned}
& N_{L 1 s t}^{\prime}\left(N_{K}\right)=\left(\begin{array}{c}
N_{M}-1 \\
N_{K}
\end{array}\right) \\
& N_{L 2 n d}^{\prime}\left(N_{K}\right)=\left(\begin{array}{c}
N_{M}-1 \\
N_{K}-1
\end{array}\right) .
\end{aligned}
$$

$N_{\text {Lrem }}$ assignments should be selected from the $N_{L}^{\prime}\left(N_{K}\right)$ assignments as the total number of assignments is $N_{\text {Lreq }}$, where $N_{\text {Lrem }}=N_{\text {Lreq }}-N_{L}\left(N_{K}-1\right) \leq N_{L}^{\prime}\left(N_{K}\right)$. When $l$ assignments are selected from the $N_{L 2 n d}^{\prime}$ assignments, the number of selection patterns is

$$
\begin{aligned}
& \left(\begin{array}{c}
N_{L 2 n d}^{\prime}\left(N_{K}\right) \\
l
\end{array}\right) \\
& \text { subject to } l \leq \min \left[N_{\text {Lrem }}, N_{L 2 n d}^{\prime}\left(N_{K}\right)\right],
\end{aligned}
$$

where $\min [a, b]$ indicates selecting the smaller value of either $a$ or $b$. Similarly, when $N_{\text {Lrem }}-l$ assignments are selected from $N_{L 1 s t}^{\prime}$ assignments, the number of selection patterns is

$$
\begin{aligned}
& \left(\begin{array}{c}
N_{L 1 s t}^{\prime}\left(N_{K}\right) \\
N_{L r e m}-l
\end{array}\right) \\
& \quad \text { subject to } l \geq \max \left[0, N_{\text {Lrem }}-N_{L 1 s t}^{\prime}\left(N_{K}\right)\right],
\end{aligned}
$$

where $\max [a, b]$ indicates selecting the larger value of either $a$ or $b$. Therefore, the average number of assignments selected from $N_{L 2 n d}^{\prime}$ assignments, i.e. $\overline{N_{L 2 n d}^{\prime}}$, is

$$
\begin{gathered}
\overline{N_{\text {L2nd }}^{\prime}}\left(N_{K}\right)=\frac{1}{N_{\text {Lall }}^{\prime}} \sum_{l=\max \left(0, N_{\text {Lrem }}-N_{L 1 s t}^{\prime}\left(N_{K}\right)\right)}^{\min \left(N_{\text {Lrem }}, N_{L 2 n d}^{\prime}\left(N_{K}\right)\right)} \\
l\left(\begin{array}{c}
N_{L 2 n d}^{\prime}\left(N_{K}\right) \\
l
\end{array}\right)\left(\begin{array}{c}
N_{L 1 s t}^{\prime}\left(N_{K}\right) \\
N_{\text {Lrem }}-l
\end{array}\right) \\
N_{\text {Lall }}^{\prime}=\left(\begin{array}{c}
N_{L}^{\prime}\left(N_{K}\right) \\
N_{\text {Lrem }}
\end{array}\right) .
\end{gathered}
$$

Similarly, the average number of assignments selected from the $N_{L 1 s t}^{\prime}$ assignments is

$$
\begin{gathered}
\overline{N_{\text {L1 st }}^{\prime}}\left(N_{K}\right)=\frac{1}{N_{\text {Lall }}^{\prime}} \sum_{l=\max \left(0, N_{\text {Lrem }}-N_{\text {L1st }}^{\prime}\left(N_{K}\right)\right)}^{\min \left(N_{\text {Lrem }}, N_{L 2 n d}^{\prime}\left(N_{K}\right)\right)} \\
\left(N_{\text {Lrem }}-l\right)\left(\begin{array}{c}
N_{L 2 n d}^{\prime}\left(N_{K}\right) \\
l
\end{array}\right)\left(\begin{array}{c}
N_{L 1 s t}^{\prime}\left(N_{K}\right) \\
N_{\text {Lrem }}-l
\end{array}\right) .
\end{gathered}
$$

4.2.3 Probabilities of RB Assignment to First- and Second-ranked MSs

Finally, the probabilities of RB assignment to the first-and second-ranked MSs are derived as follows:

$$
\begin{aligned}
& r_{1 s t}\left(N_{\text {Lreq }}\right) \\
& =\left\{\begin{array}{l}
1 \quad\left(N_{K}=0\right) \\
\frac{1}{N_{\text {Lreq }}}\left\{N_{L 1 s t}\left(N_{K}-1\right)+\overline{N_{L 1 s t}^{\prime}}\left(N_{K}\right)\right\} \quad\left(N_{K} \geq 1\right)
\end{array}\right. \\
& r_{2 n d}\left(N_{\text {Lreq }}\right) \\
& =\left\{\begin{array}{l}
0 \quad\left(N_{K}=0\right) \\
\frac{1}{N_{\text {Lreq }}}\left\{N_{L 2 n d}\left(N_{K}-1\right)+\overline{N_{L 2 n d}^{\prime}}\left(N_{K}\right)\right\} \quad\left(N_{K} \geq 1\right)
\end{array}\right.
\end{aligned}
$$

\subsection{PAPR Analysis}

The proposed technique selects one of the multiple OFDM 
frames like the SLM [7]. In the proposed technique, one or more RBs are assigned to the second-ranked MS, and the other RBs are assigned to the first-ranked MS. From [8], in the SLM, the Hadamard code sequence yields a lesser PAPR reduction than the random code sequence. One reason for this difference is the correlation between the code sequences. To extend the Hadamard code, a smaller size Hadamard code is used cyclically. As a result, the correlation between the code sequences is confirmed.

Nevertheless, this study assumes that the OFDM frame is constructed by an independent random process for simplicity of the analysis. Therefore, the analysis yields a smaller derived PAPR than the actual one.

The probability that the symbol-PAPR, i.e. $\xi_{q}$ of the $q$ th OFDM symbol $\left(q \in 0,1, \ldots, N_{Q}-1\right)$ is larger than a certain symbol-PAPR, namely $\xi_{O}$, is obtained as follows [7]:

$$
P_{S}\left(\xi_{q}>\xi_{O}\right)=1-\left(1-e^{-\xi_{O}}\right)^{N_{M} N_{D}} .
$$

The probability that the frame PAPR, i.e. $\Xi$, is larger than a certain PAPR, i.e. $\Xi_{O}$, is derived as

$$
\begin{aligned}
P_{F}\left(\Xi>\Xi_{O}\right) & =1-\prod_{q=0}^{N_{Q}-1} P_{S}\left(\xi_{q}<\Xi_{O}\right) \\
& =1-\left(1-e^{-\Xi_{O}}\right)^{N_{M} N_{D} N_{Q}} .
\end{aligned}
$$

When the number of assignment candidates is $N_{\text {Lreq }}$ and one assignment candidate is selected in a minimum PAPR manner, the occurrence probability of frame PAPR, i.e., $\Xi$, being larger than $\Xi_{O}$ is

$$
\begin{aligned}
P_{F}(\Xi> & \left.\Xi_{O}, N_{\text {Lreq }}\right)=\prod_{l=0}^{N_{\text {Lreq }}-1} P_{F}\left(\Xi>\Xi_{O}\right) \\
& =\left\{1-\left(1-e^{-\Xi_{O}}\right)^{N_{M} N_{D} N_{Q}}\right\}^{N_{\text {Lreq }}} .
\end{aligned}
$$

When the required probability is specified as $P_{L_{\text {req }}}\left(\Xi>\Xi_{O}\right)$, $\Xi_{O}$ is derived as

$$
\Xi_{O}=-\ln \left[1-\left\{1-\left(P_{L_{\text {req }}}\left(\Xi>\Xi_{O}\right)\right)^{1 / N_{\text {Lreq }}}\right\}^{1 /\left(N_{M} N_{D} N_{Q}\right)}\right] .
$$

\section{Numerical Results}

\subsection{System Configuration}

The effect of the proposed assignment is evaluated by theoretical analysis and computer simulation. Table 1 lists the evaluation parameters. For a wireless communication environment, we assume that each RB exhibits independent Rayleigh fading with flat fading within each RB. Because we assume that the time duration between the fastest and the slowest paths is smaller than the guard interval, intersymbol interference is avoidable. In this evaluation, the guard interval loss is ignored; however, if it is considered, the required
Table 1 Evaluation parameters.

\begin{tabular}{|l|l|}
\hline Wireless Access & OFDM \\
\hline Number of Subcarriers & 512 \\
\hline $\begin{array}{l}\text { Number of } \\
\text { Resource Blocks }\left(N_{M}\right)\end{array}$ & 16 \\
\hline $\begin{array}{l}\text { Number of Subcarriers per } \\
\text { one Resource Block }\left(N_{D}\right)\end{array}$ & 32 \\
\hline Size of FFT & 2048 \\
\hline $\begin{array}{l}\text { Number of OFDM symbols } \\
\text { per one frame }\left(N_{Q}\right)\end{array}$ & \begin{tabular}{l} 
(except Fig. 10$)$ \\
\hline Oversampling Factor
\end{tabular} \\
\hline Channel Estimation & Perfect \\
\hline CSI Information & Accurately Informed to BS \\
\hline Average CNR $\left(\Gamma_{O}\right)$ & 10 dB \\
\hline Error Correcting Coding & $\begin{array}{l}\text { Punctured Convolutional Code } \\
\mathrm{K}=7, \mathrm{R}=1 / 2,2 / 3,3 / 4,5 / 6\end{array}$ \\
\hline Decoder & Hard Decision Viterbi Algorithm \\
\hline
\end{tabular}

Table 2 MCS set and normalized cut-off rate.

\begin{tabular}{|c|c|c|c|}
\hline $\begin{array}{c}\text { MCS } \\
\text { No. }\end{array}$ & Modulation & $\begin{array}{c}\text { Coding } \\
\text { Rate }\end{array}$ & $\begin{array}{c}\text { Normalized Cut-Off Rate } \\
\text { (bits/symbol/subcarrier) }\end{array}$ \\
\hline 1 & BPSK & $1 / 2$ & 0.5 \\
\hline 2 & QPSK & $1 / 2$ & 1 \\
\hline 3 & QPSK & $3 / 4$ & 1.5 \\
\hline 4 & 16QAM & $1 / 2$ & 2 \\
\hline 5 & 16QAM & $3 / 4$ & 3 \\
\hline 6 & 64QAM & $2 / 3$ & 4 \\
\hline 7 & 64QAM & $5 / 6$ & 5 \\
\hline
\end{tabular}

CNR is increased by the guard interval loss.

Because the hard decision Viterbi algorithm (HDVA) is not a maximum likelihood metric and the finite-length code is used, the drop in the throughput from the Shannon's theory is carried out inevitably. Therefore, we use the throughput and cut-off rate [14] for the results of the theoretical analysis and computer simulation, respectively.

Table 2 shows the relationship between the MCS and the maximal cut-off rate performance per OFDM symbol and subcarrier. To decide the selection threshold of the MCS, we evaluate the cut-off rate performance in an AWGN channel. Figure 5 shows the results. Because we assume flat fading in each RB, the transmitter estimates the instantaneous CNR on the basis of the CSI feedback and uses it to select the most suitable MCS to maximize the cut-off rate. In this figure, we also show the throughput of Shannon's information theory [14]. We can see the performance gap between the throughput and the cut-off rate.

\subsection{CCDF of the PAPR and CDF of the Cut-off Rate}

Figure 6 shows the relationship between the frame PAPR and the complementary CDF (CCDF), i.e., $P_{F}\left(\Xi>\Xi_{O}\right)$, where the number of MSs is four. This performance is a result of a computer simulation. The performance results for $N_{\text {Lreq }}=1$ and $N_{\text {Lreq }} \neq 1$ are those without and with our proposed technique, respectively. Figure 6 shows the PAPR reduction offered by our technique. The PAPR at $N_{\text {Lreq }}=$ 512 is $2.6 \mathrm{~dB}$ smaller than that at $N_{\text {Lreq }}=1$ at a CCDF of $10^{-2}$. 


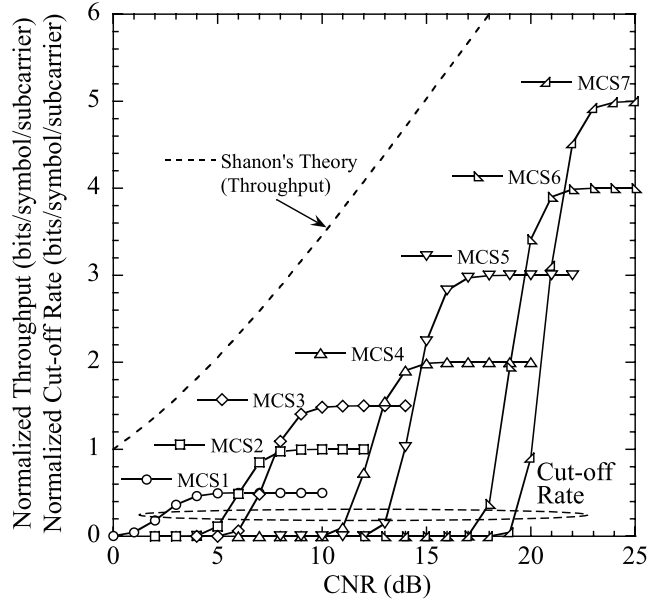

Fig. 5 Relationship between the MCS set and the CNR.

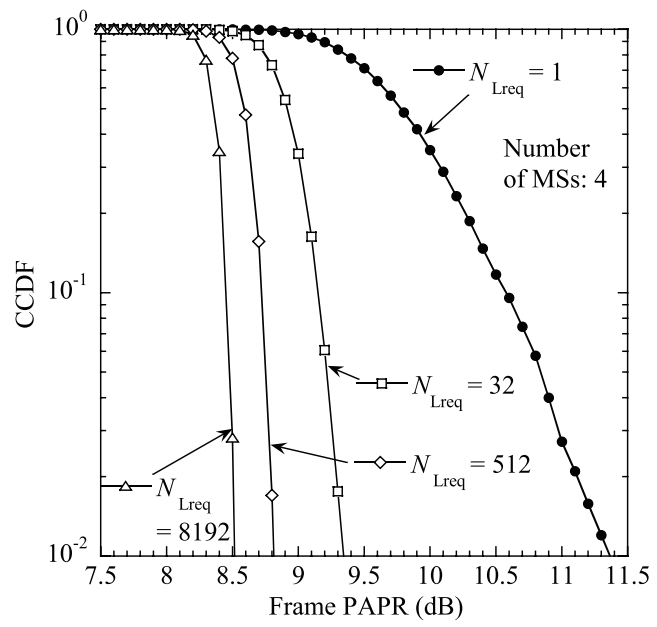

Fig. 6 CCDF performance of the frame PAPR.

Figure 7 shows the performance as expressed by the normalized cut-off rate and the CDF. In this simulation, the baseband simulation is conducted after the MCS set is selected in accordance with the instantaneous CNR. Next, each RB is checked for the bit error. If an error bit is detected, the RB is deleted, and thus, its cut-off rate is zero; otherwise, the normalized cut-off rate of the RB is that of the selected MCS set in Table 2. The obtained normalized cutoff rate for all the RBs are averaged, yielding the normalized cut-off rate per RB. Because the cut-off rates have eight patterns $(0,0.5,1,1.5,2,3,4,5$ (bits/symbol/subcarrier)), the normalized cut-off rate per subcarrier is a discrete quantity. As a result, the CDF performance is step like.

From this figure, it is observed that when $N_{\text {Lreq }}$ is large, the normalized cut-off rate is small. For comparison, when the CDF is decided, the achieved cut-off rate is evaluated. After the two-step bracketing of the CDF of interest is derived, the cut-off rate is determined by linear interpolation. For example, when the CDF of interest is 0.1 , the achieved cut-off rate at $N_{\text {Lreq }}=1$ and 512 are 1.525 and 1.460 bits/symbol/subcarrier, respectively. Therefore, the cut-off

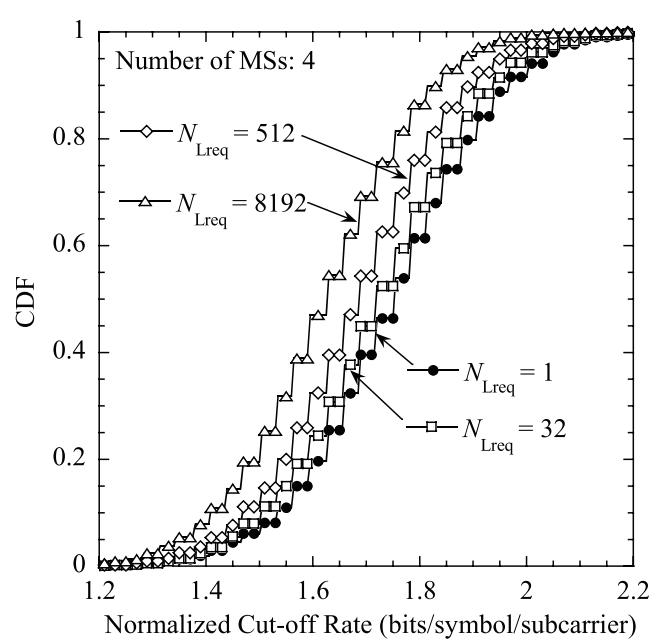

Fig. 7 CDF performance of the normalized cut-off rate.

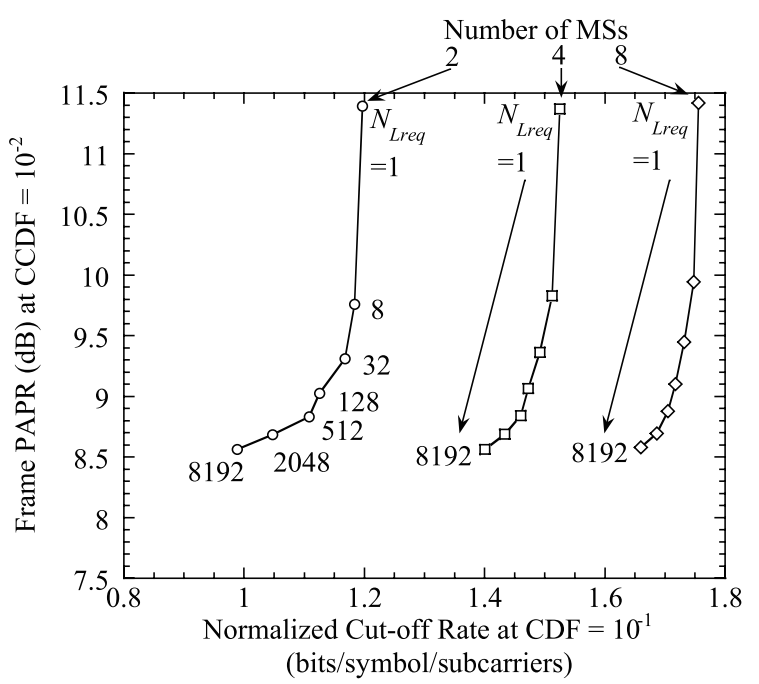

Fig. 8 Relationship between the frame PAPR at a CCDF of $10^{-2}$ and the normalized cut-off rate at a $\mathrm{CDF}$ of $10^{-1}$

rate is degraded by our proposed technique.

\subsection{PAPR-Cut-off Rate and PAPR-Throughput Tradeoffs}

Figure 8 shows the performance between the frame PAPR and the normalized cut-off rate, where the frame PAPR is evaluated at a CCDF of $10^{-2}$ and the normalized cut-off rate is evaluated at a CDF of $10^{-1}$. The numbers of MSs are two, four, and eight, and $N_{\text {Lreq }}$ is $1,8,32,128,512,2048$, and 8192.

From all results, as $N_{\text {Lreq }}$ becomes large, the frame PAPR becomes small, as well as the cut-off rate. Therefore, the cut-off rate-PAPR tradeoff set by our proposed technique can be established. In addition, the results show that as the number of MSs increases, the PAPR-cut-off rate tradeoff eases. For example, when the number of MSs is two, the cut-off rate difference between $N_{\text {Lreq }}=1$ and 8192 is $0.2 \mathrm{bits} / \mathrm{symbol} / \mathrm{subcarrier}$, but when the number of MSs is eight, the respective difference is $0.1 \mathrm{bits} / \mathrm{symbol} / \mathrm{subcarrier}$. 


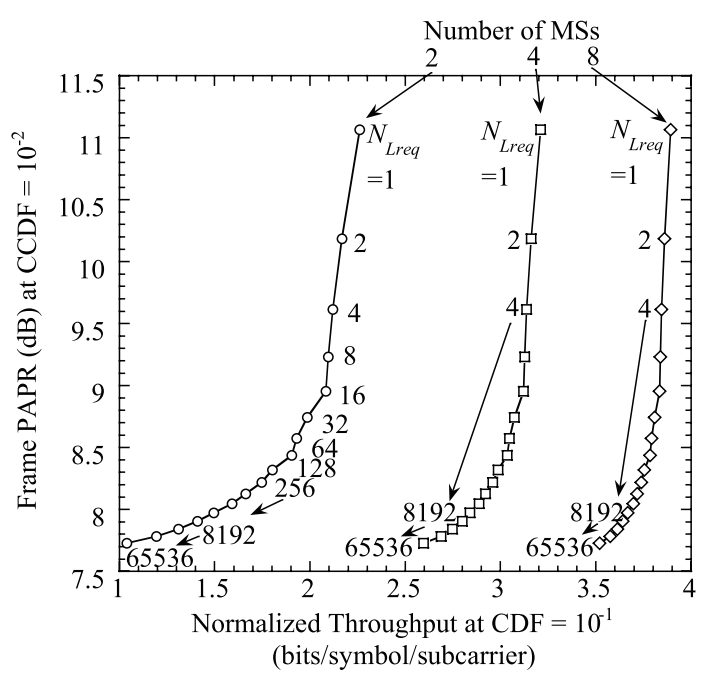

Fig. 9 Relationship between the frame PAPR at a CCDF of $10^{-2}$ and the normalized throughput at a CDF of $10^{-1}$ (theoretical analysis).

Figure 9 also shows the performance between the throughput at $\mathrm{CDF}=10^{-1}$ and the PAPR at $\mathrm{CCDF}=10^{-2}$ from the theoretical analysis, where $N_{\text {Lreq }}$ is incremented by replicating it from 1 to $65536=2^{16}$.

From the comparison between Figs. 8 and 9, the performance of the PAPR-cut-off rate derived from the computer simulation shows a similar trend to that of the PAPRthroughput derived from the theoretical analysis. Exactly, the cut-off rate is smaller than the throughput because of the use of the HDVA and the finite-length code, and a practical modulation format. In addition, the PAPR derived from the computer simulation is larger than that from the theoretical analysis because the correlation between the two assignment candidates is ignored. Thus, the PAPR reduction in the theoretical analysis is slightly overestimated.

From this figure, it is found that the throughput-PAPR tradeoff similarly eases as the number of MSs increases. For example, when the numbers of users are two and eight, the throughput differences between $N_{\text {Lreq }}=1$ and 8192 are 0.85 and 0.25 bits/symbol/subcarrier, respectively. The reason for this difference is related to the diversity limitation[10], [11]. From Eqs. (17) and (18), the diversity orders of the first-and second-ranked MSs, namely $d_{1}$ and $d_{2}$, are $N$ and $N-1$, respectively, where $N$ is the number of users. With $N=2,\left.d_{1}\right|_{N=2}=2$, and $\left.d_{2}\right|_{N=2}=1$, whereas with $N=8$, $\left.d_{1}\right|_{N=8}=8$ and $\left.d_{2}\right|_{N=8}=7$. In [10] and [11], the gap in the required energy to achieve the target bit error rate between diversity orders 1 and 2 is over $10 \mathrm{~dB}$ and that between diversity orders 7 and 8 is less than $1 \mathrm{~dB}$. Therefore, in our proposed technique, even if the RB is assigned to the second-ranked MS, when the number of MSs is large, the performance degradation is slight.

\subsection{Effect of PAPR Reduction on the Size of Frame}

Next, we consider the various number of OFDM symbols per frame. Figure 10 shows the relationship between the

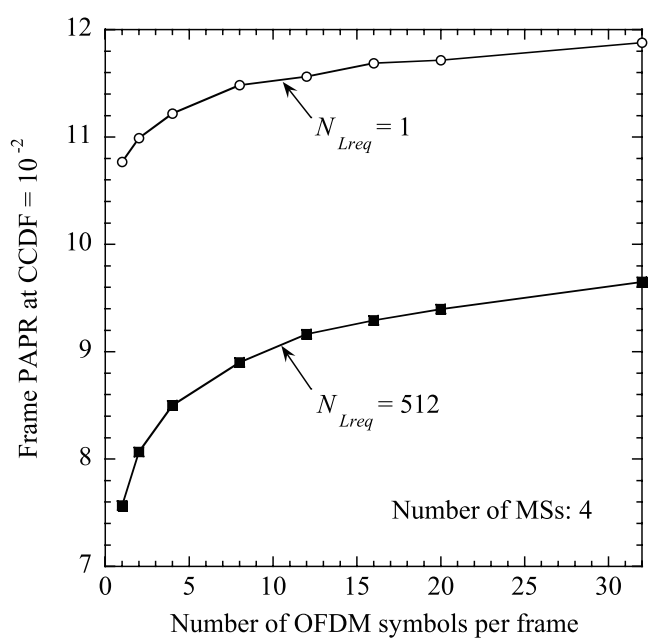

Fig. 10 Performance between the number of OFDM symbols and frame PAPR at a CCDF of $10^{-2}$

number of OFDM symbols per frame and the frame PAPR at a CCDF of $10^{-2}$, where the number of MSs is four and $N_{\text {Lreq }}$ s are 1 and 512. When the number of OFDM symbols ranges from one to four, the difference in the PAPR between $N_{\text {Lreq }}=1$ and 512 is over $2.5 \mathrm{~dB}$. When the number of OFDM symbols is greater than four, the difference is almost $2.2 \mathrm{~dB}$. From this figure, it is found that even if the number of OFDM symbols is greater than four, the proposed technique still offers some PAPR reduction.

\subsection{Results with Nonlinear Amplifier}

To investigate the effect of nonlinear distortion, the clipping model [3] is introduced as a nonlinear power amplifier, where in this model, AM/AM distortion occurs whereas the $\mathrm{AM} / \mathrm{PM}$ distortion is negligible, similar to that in a solidstate power amplifier [2]. In the clipping model, linearity is maintained up to saturation level $\Lambda$, where $\Lambda$ is normalized by the average power of the input signal.

Figure 11 shows the CCDF performance of the frame PAPR where the number of MSs is four. In the proposed technique, $N_{\text {Lreq }}$ is 512, whereas in the modified one, the target PAPR, $\Xi_{t h}$, is $8.9 \mathrm{~dB}$. Figure 11 shows at a CCDF of $10^{-3}$, the frame PAPRs of both the PAPR reduction and the PAPR reduction with $\Xi_{t h}=8.9 \mathrm{~dB}$. If the clipping level, $\Lambda$, is $8.9 \mathrm{~dB}$, the occurrence probability of the clipping nonlinear distortion is smaller than $10^{-3}$ for both the proposed technique and that with a threshold. When the frame PAPR lies in the range from $8.5 \mathrm{~dB}$ to $8.9 \mathrm{~dB}$, the CCDF of the proposed PAPR reduction with $\Xi_{t h}$ is greater than that without it, which indicates that setting the target PAPR avoids excessive downgrading of the MS rank.

To investigate the effect of the nonlinear distortion, Fig. 12 shows the performance of the frequency spectrum, where the power spectral density of each frequency spectrum is normalized by that of the desired bandwidth. Figure 12 shows one-half of the entire frequency spectra. Therefore, when the normalized frequency ranges from 0 


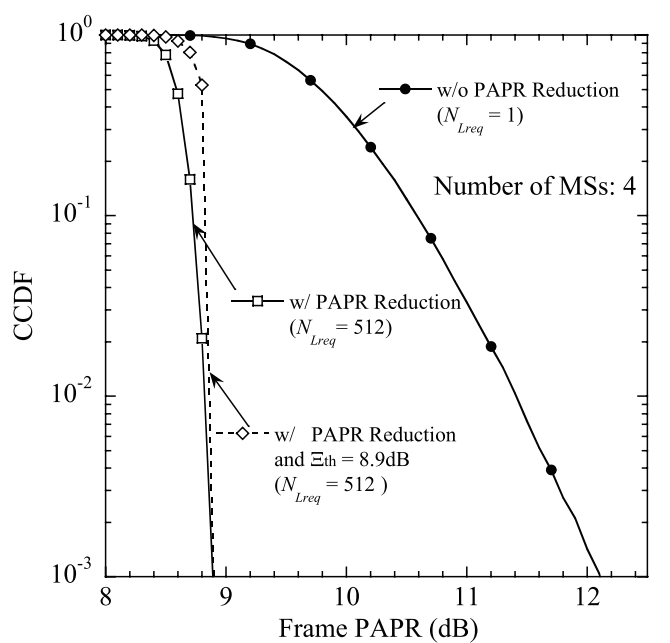

Fig. 11 CCDF performance of the frame PAPR with threshold setting.

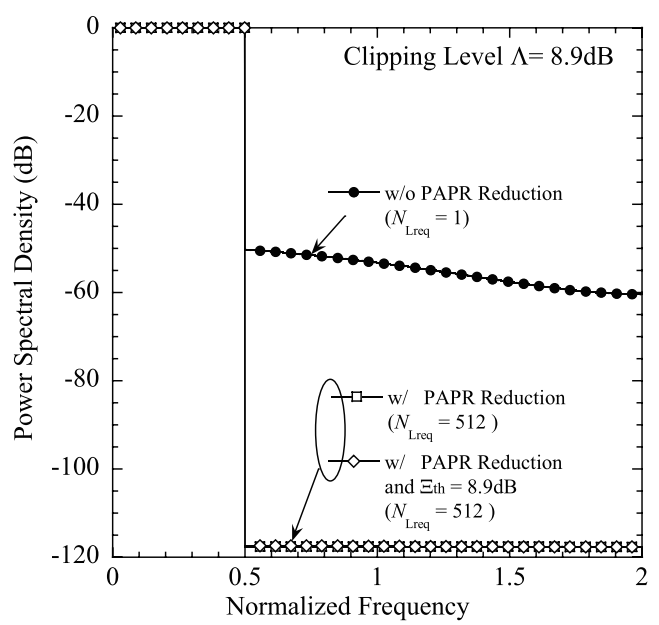

Fig. 12 Relationship between the normalized frequency and the power spectral density.

to 0.5 , power spectrum is desired; otherwise, it is an outof-band emission. This figure also shows that both the PAPR reduction technique and that with a threshold achieve $\mathrm{a}-115 \mathrm{~dB}$ in the out-of-band emission. The out-of-band emission with PAPR reduction is over $50 \mathrm{~dB}$ smaller than that without PAPR reduction. We confirm that the effect of nonlinear distortion on the OFDM frame in the PAPR reduction is at an equal level to that in the PAPR reduction with $\Xi_{t h}$.

Figure 13 shows the CDF performance of the normalized cut-off rate. "Average" and "10\%" are the results of the average normalized cut-off rate and that at the CDF of $10 \%$, respectively. The normalized cut-off rate achieved by the PAPR reduction with $\Xi_{t h}$ is slightly better than that without it. As described earlier, the former avoids excessive downgrading of the MS rank; hence, a large throughput is maintained. From these results, it is found that when the nonlinear power amplifier of the clipping model is available, using assignment with a threshold is effective to mitigate the re-

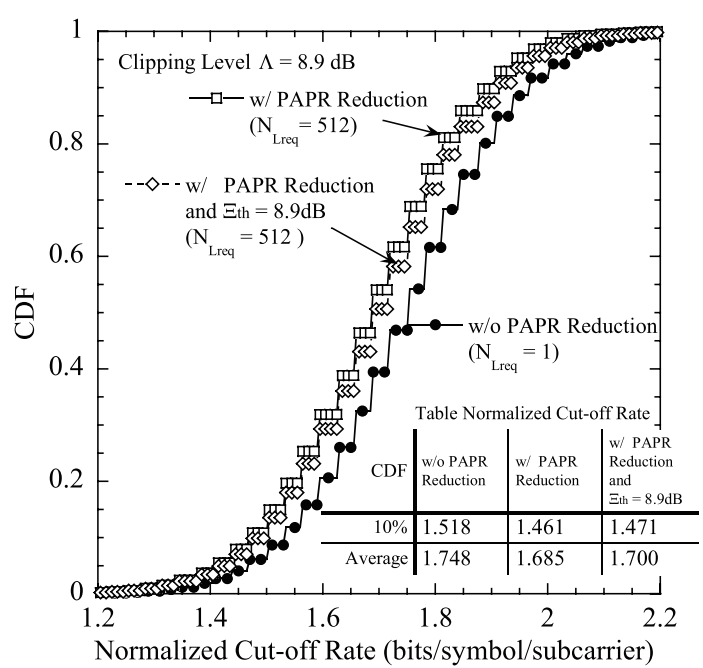

Fig. 13 CDF performance of the normalized cut-off rate.

duction in the cut-off rate and to avoid nonlinear distortion.

\section{Conclusions and Future Works}

This paper has proposed an RB assignment technique to reduce the PAPR of OFDM signals in a multiuser OFDM system. The proposed technique assigns several RBs to the second best ranked MSs if the system yields a modulated OFDM signal with reduced PAPR. Because this process degrades the diversity order by one order, the throughput is degraded with the use of our proposed technique. Therefore, we develop the PAPR-throughput tradeoff. Owing to the limitation in the diversity order, when the number of MSs is large, this tradeoff is eased. In this paper, we have also shown the tradeoff between the cut-off rate and the PAPR in a practical system by computer simulation; thus, the improvement in this tradeoff has also been confirmed.

In a cellular system, user fairness should be considered. Because each assignment candidate is almost equivalently selected, if user ranking is based on the proportional fairness criterion, our proposed technique can maintain the user fairness.

The disadvantage of the proposed technique is its large computational complexity at the transmitter. Because the PTS is similar to the proposed technique, the lowcomplexity algorithm for PTS is directly applied to the proposed technique. For example, when we use the flipping algorithm [5], the assignment of each RB to the first- or second-ranked user is decided successively. As a result, the required number of FFTs to evaluate the PAPR is $2^{16}$ in the original proposed technique, but it is only $2 \times 16$ in the proposed technique using the flipping algorithm. Other algorithms such as the genetic algorithm [16] and iterative flipping [17] are also powerful in reducing the computational complexity of the proposed technique. Therefore, it is important for our future work to find a suitable algorithm that can reduce the computational complexity of our proposed technique. 
"Clipping and filtering" is one of the important conventional techniques because it can also develop the PAPR and throughput tradeoff caused by nonlinear distortion. The powerful error-correction coding [3] can compensate for the nonlinear distortion. For comparison between the clipping and filtering and the proposed techniques, a simulator that evaluates the standardized wireless system is necessary. Therefore, performance evaluations of the proposed and the clipping and filtering techniques are also important in our future work.

\section{Acknowledgments}

A part of this research is sponsored by the Telecommunications Advancement Foundation and the Ministry of Internal Affairs and Communications in Japan as a project name of Strategic Information and Communications R\&D Promotion Programme (SCOPE 092103009) and CASIO science promotion foundation.

\section{References}

[1] C.Y. Wong, R.S. Cheng, K.B. Letaief, and R.D. Murch, "Multiuser OFDM with adaptive subcarrier, bit, and power allocation," IEEE J. Sel. Areas Commun., vol.17, no.10, pp.1747-1758, Oct. 1999.

[2] R. van Nee and R. Prasad, OFDM for wireless multimedia communications, Artech House, 2000.

[3] H. Ochiai and H. Imai, "Performance analysis of deliberately clipped OFDM signals," IEEE Trans. Commun., vol.50, no.1, pp.89-101, Jan. 2002.

[4] X. Wang, T.T. Tjhung, and C.S. Ng, "Reduction of peak-to-average power ratio of OFDM system using A companding technique," IEEE Trans. Broadcast., vol.45, no.3, pp.303-307, Sept. 1999.

[5] L.J. Cimini and N.R. Sollenberger, "Peak-to-average power ratio reduction of an OFDM signal using partial transmit sequence," IEEE Commun. Lett., vol.4, no.3, pp.86-88 March 2000.

[6] K.G. Paterson and V. Tarokh, "On the existence and construction of good codes with low peak-to-average power ratios," IEEE Trans. Inf. Theory, vol.46, no.6, pp.1974-1987, Sept. 2000.

[7] R.W. Baüml, R.F.H. Fischer, and J.B. Hüber, "Reducing the peakto-average power ratio of multicarrier modulation by selective mapping," Electron. Lett., vol.32, no.22, pp.2056-2057, Oct. 1996.

[8] N. Ohkubo and T. Ohtsuki, "Design criteria for phase sequences in selected mapping," IEICE Trans. Commun., vol.E86-B, no.9, pp.2628-2636, Sept. 2003.

[9] E. Dahlman, S. Parkvall, J. Skold, and P. Beming, 3Gevolution HSPA and LTE for mobile broadband, second ed., 2008.

[10] J. Pierce, "Theoretical limitations on frequency and time diversity for fading binary transmissions," IRE Trans. Communications Systems, vol.9, no.2, pp.186-187, June 1961.

[11] P. Hahn, "Theoretical diversity improvement in multiple frequency shift keying," IRE Trans. Commun. Syst., vol.10, no.2, pp.177-184 June 1962.

[12] Y.L. Lee, Y.H. You, W.G. Jeon, J.H. Paik, and H.K. Song, "Peak-toaverage power ratio in MIMO-OFDM systems using selective mapping," IEEE Commun. Lett., vol.7, no.12, pp.575-577, Dec. 2003.

[13] S. Haykin, Communication Systems, Wiley, 4th ed. 2002.

[14] J.G. Proakis, Digital communications, fourth ed., McGraw-hill, 2001.

[15] Z. Hongyuan, D. Huaiyu, Z. Quan, and B.L. Hughes, "On the diversity order of spatial multiplexing systems with transmit antenna selection: A geometrical approach,” IEEE Trans. Inf. Theory, vol.52, no.12, pp.5297-5311, Dec. 2006.
[16] M. Lixia and M. Murroni, "Peak-to-average power ratio reduction in multi-carrier system using genetic algorithms," IET Signal Process., vol.5, no.3, pp.356-363, June 2011.

[17] O. Takyu, T. Ohtsuki, and M. Nakagawa, "A sign selection method of orthogonal variable spreading factor code for peak power reduction in multi-rate OFCDM systems," IEICE Trans. Commun. vol.E89-B, no.4, pp.1333-1345, April 2006

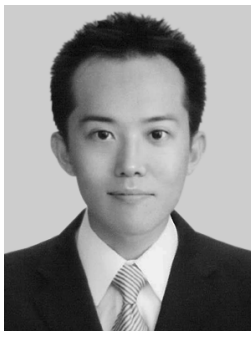

Osamu Takyu received the B.E. degree in Electrical Engineering from Tokyo University of Science, Chiba, Japan, in 2002 and the M.E. and Ph.D. degrees in Open and Environmental Systems from Keio University, Yokohama, Japan in 2003 and 2006, respectively. From 2003 to 2007, he was a research associate in the Department of Information and Computer Science, Keio University. From 2004 to 2005, he was visiting scholar in the School of Electrical and Information Engineering, University of Sydney.

From 2007 to 2011, he was an assistant professor in the Department of Electrical Engineering, Tokyo University of Science. He was an assistant professor from 2011 to 2013 and has been an associate professor from 2013 in the Department of Electrical \& Electronic Engineering, Shinshu University. Dr. Osamu TAKYU is a recipient of the Young Researcher's award of IEICE 2010 and 2010 Active Research Award in Radio Communication Systems from IEICE technical committee on RCS. His current research interests are in wireless communication systems and distributed wireless communication technology. He is a member of IEEE.

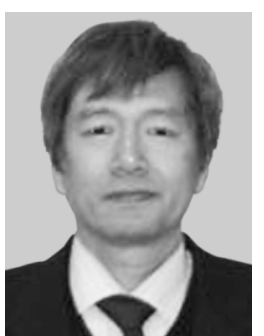

Yohtaro Umeda was born in Kanagawa, Japan, in 1957. He received the B.S. and M.S degrees in physics and the Ph.D. degree in electrical engineering from the University of Tokyo, Tokyo, Japan, in 1982, 1984, and 2000, respectively. In 1984, he joined Nippon Telegraph and Telephone (NTT) Corporation. He moved to Tokyo University of Science, Chiba, Japan, as a professor, where he is engaged in the study of high-speed analog circuits for radio and fiberoptic communication systems. Prof. Umeda is a member of the Institute of Electronics, Information and Communication Engineers (IEICE), Japan and the IEEE.

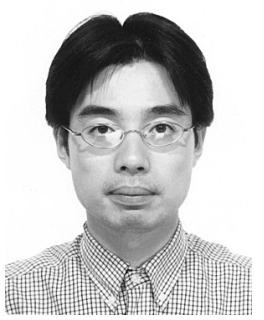

Fumihito Sasamori received the B.E., M.E and Dr.Eng. degrees from Waseda University, Tokyo in 1994, 1996 and 2000, respectively. Since 2000, he has been with the Department of Electrical and Electronic Engineering, Shinshu University, first as a Research Associate and since 2006 as an Associate Professor. His current research interests include digital mobile communication systems. He received the IEICE Young Engineer Award in 2000. He is member of IEEE. 


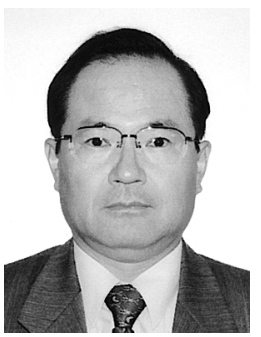

Shiro Handa received B.E. and M.E. degrees from Shinshu University in 1978 and 1980 respectively, and the Dr.Eng. degree from Kobe University in 1988. From 1982 to 1988, he was a Research Associate at Kobe University. From 1988 to 1994, he was with Nagano National College of Technology. He has been with the Department of Electrical and Electronic Engineering, Shinshu University, since 1994 as an Associate Professor and since 2005 as a professor. In 1996, he was at the University of California, Davis, as a visiting researcher. His research interests include satellite and mobile communication systems, modulation and coding, and data compression. He is a member of IEEE and SITA. 